\title{
TECNOLOGÍAS DE APOYO PARA EL FORTALECIMIENTO DE LAS \\ DESTREZAS COMUNICATIVAS EN LENGUAS EXTRANJERAS ESTUDIO DE CASO: FILMES CORTOS PRODUCIDOS POR FUTUROS DOCENTES DE INGLÉS SUPPORTED TECHNOLOGIES FOR STRENGTHENING COMMUNICATION SKILLS IN FOREIGN LANGUAGES CASE STUDY: SHORT FILMS PRODUCED BY FUTURE ENGLISH TEACHERS
}

\author{
Gonzalo Farfán ${ }^{1}$ \\ gonzalofarfan05@gmail.com \\ Jhonny Villafuerte \\ jhonny.villafuerte@gmail.com \\ Asier Romero ${ }^{2}$ \\ a.romero@ehu.es \\ Eder Intriago ${ }^{1}$ \\ eder.intriago@gmail.com
}

\author{
(1) Universidad Laica Eloy Alfaro de Manabi (Ecuador) \\ (2) Departamento de Didáctica de la Lengua y la Literatura. Bilboko Irakasleen U.E. / E.U. de Magisterio \\ de Bilbao.Euskal Herriko Unibertsitatea-Universidad del País Vasco.
}

\begin{abstract}
Resumen: Este estudio comparativo tiene como objetivo, potenciar las destrezas comunicativas: comprensión auditiva y expresión oral de futuros docentes de inglés, apoyándose en la filmación como herramienta comunicacional y didáctica. Participan 105 estudiantes de un programa de formación docente de Ecuador. El 'grupo intervención' compuesto por 50 participantes -produce filmes cortos en inglés- y mediante la observación de sus producciones detectan los errores del lenguaje cometidos, y trabajan en su auto corrección. El 'grupo control' compuesto por 55 participantes, aplica una estrategia didáctica basada en la -repetición de vocabulario- mediante aplicaciones de internet. Se administra el Knowledge English Test (KET) Cambridge Press como pre-test y pos-test para medir: corrección gramatical, entonación, pronunciación, registro lingüístico, y comprensión auditiva general y exacta. Los resultados muestran que todos los participantes mejoraron las competencias comunicativas en el segundo idioma, pero se observa mayor fluidez, claridad en las ideas expresadas, y confianza al hablar en los participantes que produjeron filmes cortos.

Palabras Claves: inglés, adquisición del leguaje, auto evaluación, educación superior.
\end{abstract}

\begin{abstract}
This comparative study aims to promote communicative skills: listening comprehension and oral expression in future English teachers, based on the filming as a communication and didactic tool. 105 students participate in a teacher training program in Ecuador. The 'intervention group' composed of 50 participants - produces short films in English - and by observing their productions participants detect the language errors committed, and work on their self-correction. The 'control group' composed of 55 participants, applies a didactic strategy based on the 'repetition of vocabulary' through internet applications. The Cambridge Press Knowledge Test (KET) is administered as a pre-test and post-test to measure: grammatical correction, intonation, pronunciation, linguistic register, and general and exact listening comprehension. The results show that all the participants improved the communicative competences in the second language, but greater fluency, clarity in the expressed ideas, and confidence in speaking on the participants who produced short films were observed.

Keywords: Language acquisition, self-evaluation, English, higher education.

aprendizaje ricos en tecnología, enseñanza.
\end{abstract}

$-183-$

Píxel-Bit. Revista de Medios y Educación. No 51 Julio 2017. ISSN: 1133-8482. e-ISSN: 2171-7966. doi: http://dx.doi.org/10.12795/pixelbit.2017.i51.12 


\section{Introducción.}

Finalizada la aplicación de pruebas estandarizadas de conocimiento del idioma en Ecuador en el año 2012, se determinó que solamente $4 \%$ de los 5.000 evaluados lograron alcanzar el nivel B2 del Marco Común Europeo de Referencia para las Lenguas (British Council, 2015) requerido para ingresar al magisterio nacional en calidad de docente de lengua extranjera a tenor del marco legislativo: Orgánica de Educación Superior (República de Ecuador, 2010) y Orgánica de Educación Intercultural (República de Ecuador, 2011). Así, entre las medidas implementadas por el gobierno ecuatoriano, se puso en marcha el programa GO TEACHER, con el objetivo de que los profesores alcancen el nivel de perfeccionamiento de las Segunda Lengua (L2) en países anglosajones desde el año 2013. Estos esfuerzos han dado frutos importantes, pero la demanda nacional de docentes de inglés que supera los 4.000 profesionales, no ha sido cubierta en su totalidad (República de Ecuador, 2015). Esta circunstancia motivo la implementación en el año 2016, de un programa de voluntariado para que profesionales nativos de habla inglesa, participen del proyecto de enseñanza masificada en Ecuador de manera temporal. Además, se demanda a la academia ecuatoriana la implementación a mediano plazo de programas de formación docente que garanticen el cumplimiento de los actuales estándares internacionales en el campo de las lenguas extranjeras.

A pesar de que la integración intensiva y extensiva de las Tecnologías de la Información y Comunicación (TIC) en los sistemas de educación en los diversos países del mundo, ha dependido de la disponibilidad de los medios necesarios, nunca antes las tecnolo- gías habían estado tan involucradas en el proceso de enseñanza y aprendizaje de la población (Cabero, Llorente, y Gómez del Castillo, 2011). Por lo tanto, las comunidades educativas requieren de docentes de vanguardia dotados de competencias digitales que les permita potenciar su auto-formación, obtener datos actualizados para mejorar la calidad de la enseñanza (Vera, 2012), y hacer de su alumnado, participes de procesos de intercambio cultural a nivel internacional; ya que la educación mediática ha demostrado ser capaz de promover la cultura participativa y la autonomía en los aprendientes (UNESCO, 2008; Esteve, 2009; Ferrés \& Piscitelli, 2012).

Ante el reto de adquirir una lengua extranjera se requiere de la 'motivación', que descrita por Touré-Tillery y Fishbach (2014), es aquella «fuerza psicológica que lleva a la acción» (p. 328), siendo uno de los factores principales que anima a las personas a dirigir esfuerzos y emprender rutas diversas para alcanzar niveles de dominio de una lengua extranjera. La clase de inglés en todos los niveles del sistema educativo requiere de didácticas y herramientas que potencien los logros del alumnado. Así, encuentra en la producción de filmes cortos y el apoyo de herramientas computacionales, los recursos que tienen el poder de captar la atención del aprendiente, generar practicas idiomáticas, y motivar su aprendizaje (Quesada, 2015). Por su parte, los aprendientes han sido motivados a incorporar a su repertorio de competencias el uso las TIC Cevallos, Intriago, Villafuerte, Molina, y Ortega (2017), destreza que es necesario aprovechar, para aportar al mejoramiento del sistema educativo al inicio del siglo XXI.

Las destrezas de interacción oral en el uso de una lengua extranjera: comprensión auditiva y expresión oral, retoman su

- 184 -

Píxel-Bit. Revista de Medios y Educación. No 51 Julio 2017. ISSN: 1133-8482. e-ISSN: 2171-7966. doi: http://dx.doi.org/10.12795/pixelbit.2017.i51.12 
importancia en la comunicación humana (Etxebarria, Garay y Romero (2012), y su calidad es fundamental para la emisión y recepción clara de los mensajes; ya que la semántica, ambivalencia y significado de los términos (Fernández, 2009) deben ser aplicados de manera correcta.

Adicionalmente, la adquisición y dominio de una lengua se vincula al contexto en el que se encuentra sumergido el aprendiente (Saravia y Bernaus, 2008; Etxebarria, Garay, y Romero, 2012), y que se caracteriza por el trabajo en equipo a través de redes sociales (Cabero \& Marín, 2013), y mediante procesos que articulan los valores e intereses humanos con el uso de las TIC, a partir del reconocimiento que el «construccionismo social se manifiesta en diferentes ámbitos de la vida social, como es el caso de la memoria, las conversaciones, las interacciones y la educación» (Mendoza, 2015, p. 86), y de manera general, el alumnado ha alcanzado altos como utilitarios de los dispositivos electrónicos (García-Salinas, Ferreira-Cabrera, y Morales-Ríos, 2012; Vera, 2012; Quesada, 2015; Cevallos, Intriago, Villafuerte, Molina, \& Ortega, 2017), pero requieren del apoyo didáctico para trabajar en el análisis de la información disponible.

En el caso de Ecuador, según Sarmiento (2009), se deben mantener los esfuerzos para mejorar y diversificar el componente pedagógico en relación al uso de las TIC; y ampliar la cobertura para el trabajo con diversas edades; tomando ventaja de su clara capacidad de atracción, intereses individuales, etc. (Intriago, Villafuerte, Morales, Lema, \& Echeverría, 2016); y a partir de las acciones curriculares centradas en el alumno, en las que se estimula en los aprendientes la toma de decisiones para fortalecer las «capacidades para organizar, dirigir, monitorear y evaluar su aprendizaje» (Hernández e Izquierdo, 2016, p.48).

En el escenario descrito, este trabajo pretende contribuir a los procesos de construcción del conocimiento en el área de lenguas extranjeras en el marco de los retos nacionales, potenciando la comprensión auditiva y expresión oral del inglés en futuros docentes de Ecuador, mediante el uso de la filmación como herramienta comunicacional y didáctica. Por tanto, las interrogantes de investigación planteadas son: ¿Cuál es la contribución de la herramienta filmación en procesos de adquisición de una lengua extranjera? ¿Es posible auto detectar los errores cometidos en la comprensión auditiva y la expresión oral a través de la producción de filmes cortos? ¿En qué medida la producción de filmes cortos logra generar espacios de intercambio e interacción social dando uso a una lengua extranjera?

\section{Revisión Literaria.}

\subsection{Tecnologías de apoyo en la educación del siglo XXI.}

A criterio de Boyd y Ellison (2007), las TIC facilitan el intercambio de datos entre aquellos que comparten una conexión, recorren una lista de conexiones de otros miembros del sistema, y aportan a la generación de una comunidad virtual especializada en la producción de imágenes, fotografías y multimedia. Según Cabero, Llorente, y Gómez (2011), una de las metas al usar las tecnologías de apoyo es procurar que los estudiantes tomen conciencia de la realidad y obtengan aprendizajes por medio de la comunicación social con sus iguales.

Según Vera (2012), el termino TIC fue definido por el Programa de las Naciones

$-185-$

Píxel-Bit. Revista de Medios y Educación. No 51 Julio 2017. ISSN: 1133-8482. e-ISSN: 2171-7966. doi: http://dx.doi.org/10.12795/pixelbit.2017.i51.12 
Unidas para el Desarrollo (PNUD) como un «universo de dos conjuntos». De allí que, por un lado, las 'tecnologías de la comunicación’ (TC) (radio, televisión, la telefonía convencional, etc.) se unen a las 'tecnologías de la información' (dispositivos inteligentes, internet, software, etc.) (TI) para el registro de los contenidos aplicados a procesos educativos.

Así, las TIC son un conjunto de servicios, redes, software, y aparatos que tienen como fin la mejora de la calidad de vida dentro de un entorno, integrándose a un sistema de información interconectado y complementario» (Vera, 2012, p. 2).

\subsection{La filmación como herramienta didáctica.}

El uso de la filmación y el video en procesos de enseñanza y aprendizaje del idioma inglés en estudiantes universitarios, apoya la tendencia de reducción gradual del soporte a los medios analógicos y el aumento del aprendizaje digital. Más allá de los aspectos técnicos como decibeles, pixeles, gigas, voltios, etc., expresados en sonido, imágenes, velocidad e iluminación, un 'filme corto' que se apoya en las tecnologías tiene un lenguaje propio, cuya secuencia induce al receptor a sintetizar sentimientos, ideas, concepciones, etc.; siendo capaz de reforzar o modificar aquellos que la persona ya tenía como acumulado de un aprendizaje previo (Monteagudo-Valdivia, 2007). Así, los productos obtenidos de forma participativa son los libretos, planes de logística, escenografía, el video y su edición (Berk. 2009). Mirados desde el enfoque educativo, los videos tienen la capacidad de «metodizar actuaciones y enfoques, profundizar el uso de técnicas, recompensar y sintetizar acciones y reacciones, al igual que captar y reproducir situaciones reales excepcionales, que pueden ser analizadas minuciosamente en diferentes momentos» (Monteagudo-Valdivia, 2007, p 2), pudiendo ser utilizados como evidencias de evaluación y ejemplificación. Los estudiantes son capaces de trabajar con sofisticados y abundantes recursos de video y audio (Temprano, 2011) acercándose al contexto de los países de habla inglesa; y que también aportan en su formación vocacional (Fernández y Torres, 2015); y los productos generados pueden ser compartidos a través de las redes sociales y otros servicios en línea tales como YouTube, Facebook, WhatsApp, etc.

La imagen en movimiento a través de las pantallas tiene tal poder de atracción que cautiva a los observadores, ya sea en formato de video, juego, programa de televisión, video musical, película, etc., (Quesada, 2015). Sin embargo, toda intervención educativa que incluya actividades artísticas y creativas para el desarrollo de las destrezas idiomáticas debe garantizar «al estudiante estar consciente de los objetivos y alcances de tales secuencias, favoreciendo la toma de decisiones y monitoreo de sus fortalezas y necesidades para lograr el dominio de la L2» (Hernández \& Izquierdo, 2016, p.49).

\subsection{El construccionismo social y la adquisición de lenguas extranjeras.}

El lenguaje en su forma de 'diálogo' conserva aquel poder para lograr despertar el 'pensamiento' en los estudiantes, motivarles y animarles en la ruta del aprendizaje (Saravia \& Bernaus, 2008); pero, el desarrollo tecnológico y aspectos socioculturales tales como la globalización, cambio climático, consumismo, medios de

- $186-$

Píxel-Bit. Revista de Medios y Educación. No 51 Julio 2017. ISSN: 1133-8482. e-ISSN: 2171-7966. doi: http://dx.doi.org/10.12795/pixelbit.2017.i51.12 


\begin{tabular}{lcccccc}
\hline & \multicolumn{2}{c}{ Grupo control } & \multicolumn{2}{c}{ Grupo intervención } & \\
& Periodo & Femenino & Masculino & Femenino & Masculino & Total \\
\hline 2014 & 11 & 12 & 12 & 7 & 42 \\
2015 & 12 & 6 & 11 & 11 & 40 \\
2016 & 8 & 6 & 7 & 2 & 23 \\
\hline Total, según sexo & 31 & 24 & 30 & 20 & 105 \\
\hline Total, según grupos & & 55 & & & 50 & \\
\hline
\end{tabular}

Tabla 1. Muestra de participantes en la intervención. Fuente: Registros en Secretaria ULEAM.

comunicación, entre otros factores, generan nuevos escenarios, $\mathrm{y}$, por tanto, los intereses y preferencias de los aprendientes cambian permanentemente. A este punto, el avance tecnológico pone al servicio de la construcción colectiva del conocimiento, el valor añadido de los medios interactivos y espacios virtuales que apoyan nuevas formas de dialogo a través de redes sociales, correo electrónico, video, etc., y que dan paso a la educación del siglo XXI a través del modelo de formación a distancia y la educación 'digital' donde la creación colectiva, el intercambio de recursos y el control de la calidad de forma colaborativa (Ribes, 2007) logran transmitir la individualidad, personalidad, medio social, y los valores de los participantes (Saravia y Bernaus, 2008).

Si bien es cierto, la visión del conocimiento compartido, dedica especial atención a las «transacciones conversacionales» pero, también reconoce la existencia de otras formas de edificar el conocimiento tales como «prácticas sociales, percepciones, grafos, y otras manifestaciones de interacción social» (Sisto, 2012, p. 3.), y dónde el 'socio construccionismo' conserva disciplinas tales como antropología, sociología, y psicología social, e incluso en la psicología para promover el diálogo en torno a las personas y sociedades. Por lo tanto, el lenguaje tiene un papel protagónico en la construcción social del conocimiento, ya que se trata del canal necesario para su realización.

Haciendo uso del lenguaje, las personas elaboran preguntas respecto a asuntos que ignoran, pero de la misma manera reciben las reacciones respecto a lo que la gente conoce o domina. De esta forma, al unir el conocimiento y la realidad, el lenguaje alcanza aquel nivel preponderado que Sisto (2012) define como la 'Segunda Revolución Cognitiva'. No obstante, la construcción del conocimiento en las aulas universitarias corre el riesgo de debilitarse, cuando el diálogo deja de producirse como efecto de una falta de estimulación adecuada y pertinente; Así, Mendoza (Mendoza, 2015) recalca el valor de los «intercambios verbales [...] como forma de interacción para el logro del conocimiento» en el contexto del aula (p. 87).

Entre estudios previos revisados aparece el trabajo realizado por Sarmiento (2009) en Ecuador, respecto al uso de TIC y la medición de la competencia digital en estudiantes de secundaria. Sus conclusiones indican que la contribución de las TIC supera la eficiencia lingüística funcional, y aporta a la habilidad de construir y reconstruir nuevos conocimientos durante la comunicación en una lengua extranjera.

Otro trabajo relevante es el que ejecutaron Garay, Luján y Etxebarria (2013); quienes

$-187-$

Píxel-Bit. Revista de Medios y Educación. No 51 Julio 2017. ISSN: 1133-8482. e-ISSN: 2171-7966. doi: http://dx.doi.org/10.12795/pixelbit.2017.i51.12 
Comprensión auditiva (promedio entre comprensión general y exacta)

\begin{tabular}{cccccccc}
\hline \multicolumn{7}{c}{ MARCO COMÚN EUROPEO DE REFERENCIA DE LA LENGUA (MCER) } & Total, \\
Grupos & C2 & C1 & B2 & B1 & A2 & A1 & participantes \\
\hline Intervención & 0 & 0 & 0 & 10 & 35 & 5 & $\mathbf{5 0}$ \\
Control & 0 & 0 & 0 & 13 & 34 & 8 & 55 \\
\hline Expresión oral (entonación, fluidez y registro aplicado) & & & & \\
\hline Intervención & 0 & 0 & 1 & 15 & 29 & 5 & 50 \\
Control & 0 & 0 & 3 & 16 & 28 & 8 & $\mathbf{5 5}$ \\
\hline
\end{tabular}

Tabla 2. Resultados pre-test en comprensión auditiva y expresión oral

Fuente: Registro de las pruebas KET Cambridge aplicadas (2014, 2015 y 2016)

compararon las destrezas que tiene el alumnado de dos universidades españolas respecto al uso académico de instrumentos de la Web 2.0. En dicho estudio se constata la eficiencia de los recursos tecnológicos ofrecidos para asimilar, afianzar y reflexionar sobre los contenidos aprendidos.

Estudios de Hernández e Izquierdo (2016) respecto al fortalecimiento de la expresión oral en L2 demuestran que, «en la práctica docente, el proceso de la escucha: preescucha, escucha, y pos-escucha deben ser acompañados por objetivos», que aporten con claridad al mejoramiento de las destrezas comunicativas.

\section{Metodología.}

Este trabajo de investigación acción acude a las técnicas cuantitativas para comparar los resultados sobre las variables: comprensión auditiva y expresión oral en idioma inglés, aplicado a un grupo de estudiantes universitarios domiciliados en Ecuador.

Este es un estudio comparativo aplicado durante 3 años (periodo 2014-2016) y cuando los participantes tomaron la asignatura: inglés para fines específicos; es decir durante el séptimo semestre de un total de nueve semestres.

La muestra

La muestra la componen 105 estudiantes del Programa Licenciatura en Idiomas, Mención inglés (futuros docentes de inglés) de la universidad Laica Eloy Alfaro de Manabí. Ver tabla 1.

Se trata de una muestra heterogénea compuesta por un acumulado de 60 personas de sexo femenino y 44 de sexo masculino, en edades comprendidas entre 21 y 45 años.

Los instrumentos

Los instrumentos utilizados son:

a. Fichas de datos generales de los participantes en las que se incluyen datos personales y record académico.

b. Para la administración del Pre-test y Post-test se aplica la prueba de conocimiento KET Cambridge Press (Knowledge English Test); instrumento aplicado internacionalmente, y cuya validez y confiabilidad está dada por Cambridge Press.

La propuesta didáctica:

El proceso compara dos tipos de estrategias didácticas para la adquisición de lenguas extranjeras que son ejecutadas desde un grupo intervención (50 participantes) y un grupo control (55 participantes).

$$
-188-
$$


Comprensión auditiva (promedio entre comprensión general y exacta)

\begin{tabular}{cccccccc}
\hline \multicolumn{7}{c}{ MARCO COMÚN EUROPEO DE REFERENCIA DE LA LENGUA (MCER) } & $\begin{array}{c}\text { Total, } \\
\text { Grupos }\end{array}$ \\
C2 & C1 & B2 & B1 & A2 & A1 & participantes \\
\hline Intervención & 0 & 0 & 0 & 29 & 21 & 0 & $\mathbf{5 0}$ \\
Control & 0 & 0 & 0 & 18 & 37 & 0 & $\mathbf{5 5}$ \\
\hline Expresión oral (entonación, fluidez y registro aplicado) & & & & \\
\hline Intervención & 0 & 0 & 5 & 35 & 10 & 0 & $\mathbf{5 0}$ \\
Control & 0 & 0 & 3 & 20 & 32 & 0 & $\mathbf{5 5}$ \\
\hline
\end{tabular}

Tabla 3. Resultados pos-test en comprensión auditiva y expresión oral

Fuente: Registro de las pruebas KET Cambridge aplicadas (2014, 2015 y 2016)

El grupo intervención participa de actividades metacognitivas esperando que estas activen y afinen en los participantes la capacidad para identificar los errores que comenten al escuchar y hablar en la lengua extranjera. Como instrumento de autoevaluación se aplican los filmes cortos. Los participantes aplican las destrezas de comunicación en idioma inglés que poseen para cursar por las etapas de creación, producción y actuación en las filmaciones. Se comunican en idioma inglés con sus colegas y facilitadores el mayor tiempo posible para lo que es necesario asumir una posición de compromiso personal con el proceso. Se implementan sobre la agenda momentos para la autoevaluación, reflexión y autocorrección, y para lo que se pone a disposición el soporte del profesor de la asignatura inglés para fines específicos y soporte técnico para el uso de los equipos tecnológicos. En esta etapa se siguió la metodología que Berk propuso en el año 2009, en la publicación titulada «Multimedia teaching with video clips: TV, movies, YouTube, and Mtv.». Lógicamente, esta metodología fue implementada atendiendo al contexto local. Las actividades fueron: selección del tema y género de la filmación (terror, acción, drama, romance, etc.); Asignación de los papeles en el filme y en la producción (guionistas, actores y actrices, maquillistas, etc.); creación del libreto; creación de la escenografía y selección de sonido; primera filmación; taller meta cognitivo 1; segunda filmación; taller meta cognitivo 2; edición. Finalmente, a estos pasos se agregó el pre-test y los pos-test aplicados al inicio y fin de la intervención.El grupo control -acompañado por un facilitador- ejecutó ejercicios de repetición de palabras nuevas para agregarlas al vocabulario, se ejercitaron de forma individual durante sus tiempos libres y no participaron de actividades grupales ni de procesos metacognitivos bajo supervisión. Los participantes del grupo control tuvieron la libertad para aplicar otras estrategias que ellos consideraron les había dado buenos resultados en prácticas anteriores. Los miembros del grupo control participaron de las pruebas pre-test y pos-test aplicados al inicio y fin de cada periodo de intervención.

- $189-$

Píxel-Bit. Revista de Medios y Educación. $N^{o} 51$ Julio 2017. ISSN: 1133-8482. e-ISSN: 2171-7966. doi: http://dx.doi.org/10.12795/pixelbit.2017.i51.12 


\section{Resultados.}

Sección 1: Los resultados del Pre-test y de Pos-test

A continuación, se exponen los resultados de las mediciones cuantitativas aplicadas a todos los participantes sobre las variables comprensión auditiva (general y exacta) y sobre la expresión oral (entonación, fluidez y registro aplicado). Ver tablas 2 y 3 .

Los resultados indican que la mayoría de los participantes en ambos grupos se ubican, tanto para la comprensión auditiva como para la expresión oral en inglés, en el nivel A2 (básico alto); existe mejor desarrollo de la expresión oral, ya que algunos participantes reportan el nivel B1 (intermedio bajo) y B2 (intermedio alto); en comparación con la comprensión auditiva.

Los resultados indican que los participantes culminan el proceso manteniendo mayor desarrollo de la destreza comunicativa 'expresión oral' de manera especial en el -grupo intervención- donde se incrementó el número de participantes a 5 en nivel B2, 35 en el nivel B2 (intermedio alto); $y$ 10 en el nivel B1. En comparación con el grupo control en la que se mantienen 3 participantes en el nivel B2, el nivel B1 se incrementa a 20, y el nivel A2 en 32 participantes.

Los mayores resultados tanto en el 'grupo intervención' como en el 'grupo control' se observan a nivel de los estudiantes que tenían menor nivel y que en el pre-test reportaron A1; ya que en el pos-test alcanzaron el nivel A2 en ambos grupos.

Finalmente, llama la atención que en el 'grupo control' no se observan avances significativos en las posiciones B2., de mayor nivel de la escala MCERL.

Sección 2: Avances dentro del grupo intervención

De manera general se observa un avance en la expresión oral de los participantes. Así, en el Pre-test expresado en color azul, los resultados obtenidos se concentran en el nivel A2 (básico alto) de la escala MCER También, se observan resultados en el nivel B1 (intermedio) y otros A1 (básico). Ver figura 1 y 2 .

En el pos-test expresado en color rojo, el resultado se concentra en $\mathrm{B} 1$ (intermedio) y A2 (básico alto); siendo el mayor logro para los estudiantes que estaban en nivel

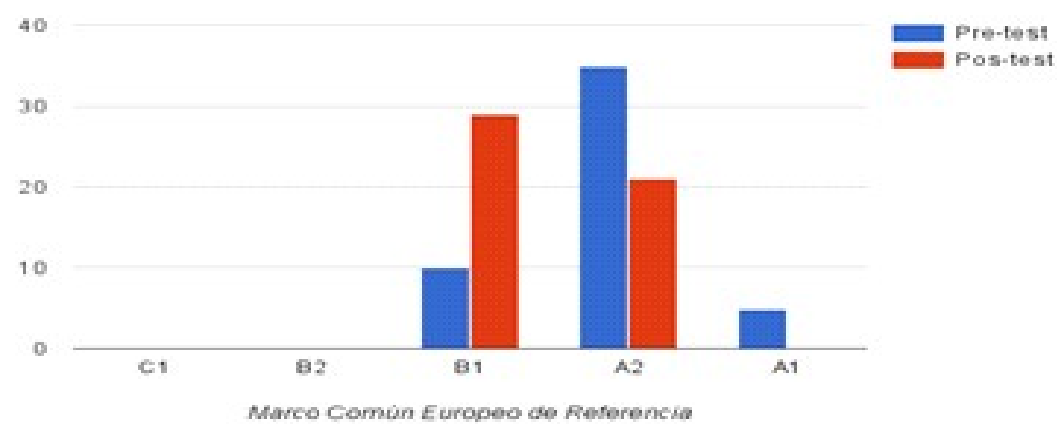

Figura 1. Avances del grupo intervención en comprensión auditiva del inglés

$-190-$

Píxel-Bit. Revista de Medios y Educación. No 51 Julio 2017. ISSN: 1133-8482. e-ISSN: 2171-7966. doi: http://dx.doi.org/10.12795/pixelbit.2017.i51.12 


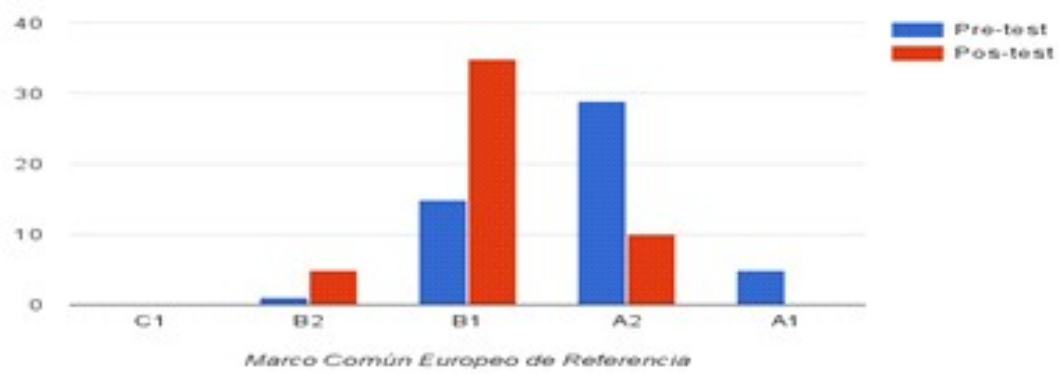

Figura 2. Avances del grupo intervención en expresión oral en inglés

A1(básico), y que además, alcanzaron un mejor nivel al final del proceso.

En cuanto a los resultados alcanzados por los participantes en la destreza expresión oral, se observa que los estudiantes más dedicados a la práctica idiomática alcanzaron el nivel B2 del MCER, el que es el nivel más alto esperado por los investigadores en esta intervención educativa.

\section{Discusión.}

Este trabajo evalúa los logros obtenidos por dos estrategias de adquisición de lenguas extranjeras apoyadas en herramientas informáticas (producción de filmes cortos, y repetición de vocabulario desde aplicaciones de internet). Al ser los filmes cortos productos de la creatividad de los participantes, se ingresa en el campo del proceso de aprendizaje como un acto propio (GarcíaSalinas, Ferreira-Cabrera, \& Morales-Ríos, 2012) individual y colectivo, que en relación a las motivaciones de los participantes podrían ser de carácter intrínseco para unas personas, o de carácter extrínseco en otras (Saravia \& Bernaus, 2008; Cevallos, Intriago, Villafuerte, et al, 2017). Las practicas idiomáticas seleccionadas en este trabajo promueve en los participantes, el uso de expresión oral y el fortalecimiento de estructura gramatical, fluidez, coherencia contextual y uso del registro apropiado (Fernández, 2009; Etxebarria, Garay \& Romero, 2012).

En el grupo intervención, los 'personajes de actuación y roles de trabajo' que cada uno de los participantes eligió, fueron observados para evaluar las destrezas comunicativas empleadas. Se observa que los personajes reflejan cualidades correspondientes a la personalidad propia y edad (formas de saludar, gestos y otras posiciones corporales, etc.) situación que es coherente en lo referente a la influencia del contexto con lo expuesto por Saravia y Bernaus, 2008, Además, se da la circunstancia de que se trata de estudiantes que no han sido entrenados como actores profesionales. Finalmente, el proceso en las diferentes etapas (creación, producción y edición) generó experiencias significativas respecto al uso correcto del idioma inglés, pero también como un proceso compartido y dentro de una atmósfera de respeto, los participantes crearon un producto inédito, y tomaron decisiones para cumplir con el proyecto en los tiempos previstos.

- $191-$

Píxel-Bit. Revista de Medios y Educación. No 51 Julio 2017. ISSN: 1133-8482. e-ISSN: 2171-7966. doi: http://dx.doi.org/10.12795/pixelbit.2017.i51.12 
En el grupo control se aplicó el método 'repetición de palabras nuevas a través de aplicaciones disponibles en internet'. Se debe notar que los participantes intencionalmente, no fueron inducidos a un trabajo colaborativo. No se propiciaron desde la intervención educativa oportunidades para el intercambio de información y otras actividades de carácter colaborativo. Se dejó a cada participante en libertad para que cada uno localice por su cuenta al menos una técnica de estudio, pero siempre con la promoción del empleo de tecnología educativa. Se remarca que los participantes recibieron por parte del facilitador, la sugerencia de trabajar en la detección de errores cuando hacen uso del idioma extranjero, y de trabajar en las correcciones que consideren necesarias. Ahora bien, en todo momento se dejó que ellos tomen libremente iniciativas para implementarlas en su proceso de conocimiento. Se esperaba el surgimiento espontáneo de iniciativas de colaboración; sin embargo, se observó el surgimiento de una dinámica competidora para lograr alcanzar el que mayor número de palabras aprendidas. Se agrega que los participantes acumularon nuevo vocabulario, lo que favoreció el avance en la adquisición del L2, pero los avances de la expresión oral fueron escasos.

Los participantes hicieron uso de las TIC aplicando una amplia gama de ejercicios obtenidos en diferentes aplicaciones disponibles en internet. Sin embargo, no fueron acompañados de manera específica, y los resultados ratifican la posición de autores como Esteve (2009); Cabero y Marín (2013); Intriago, Villafuerte, Morales, et al (2016); cuando afirman que los usos de las TIC requieren de un plan didáctico con objetivos claros para lograr su máximo beneficio. Desde la vertiente docente, esta experiencia reafirma la posición de Hernández e Izquierdo (2016) cuando argumentan que el «docente requiere de una planeación reflexiva que le permita crear secuencias didácticas fundamentadas en los procesos que caracterizan el desarrollo de una lengua adicional» (p.49).

Finalmente, los resultados indican que la producción de filmes cortos como práctica comunicacional y didáctica, ofreció a los participantes la oportunidad de autoevaluarse, plantear rutas de mejoramiento de la comprensión auditiva y expresión oral, logrando un avance de las destrezas cognitivas en aproximadamente un $30 \%$ mayor en comparación al grupo control. Se destaca que al final de la intervención educativa, se observa en el "grupo intervención» una mayor fluidez, naturalidad y confianza en el momento de hacer uso del L2.

\section{Conclusiones.}

Más allá de lograr fortalecer las destrezas comunicativas en los participantes, este trabajo pretender aportar con estrategias cognitivas dirigidas a la práctica docente y con pautas para la verbalización y reflexión en los futuros docentes de idiomas, el reconocimiento de las fortalezas y debilidades, propias y del alumnado, en el momento de hacer uso de una lengua extranjera.

Se reafirma la capacidad que tiene las TIC como herramienta comunicacional y didáctica capaz de potenciar los procesos de aprendizaje (Cabero \& Castaño, 2009), y que en el campo de la adquisición de una segunda lengua (L2) tiene usos diversos (GarcíaSalinas, Ferreira, \& Morales, 2012; Intriago, Villafuerte, Morales, et al. 2016).

- 192 -

Píxel-Bit. Revista de Medios y Educación. $N^{o} 51$ Julio 2017. ISSN: 1133-8482. e-ISSN: 2171-7966. doi: http://dx.doi.org/10.12795/pixelbit.2017.i51.12 
La producción de filmes cortos de las practicas idiomáticas se constituyen en evidencias que integran imágenes en movimiento y sonidos reales, emitidos por los aprendientes, herramientas que deben ser utilizadas en procesos de autoevaluación, evaluación par y corrección, para el fortalecimiento de las destrezas comunicativas en el uso de L2.

Para el éxito de las intervenciones de este tipo, se recomienda establecer desde la etapa de inicio, pactos de compromiso, cooperación y respeto entre los participantes. Además, el profesorado debe contar con bagaje teóricoempírico con respecto a las estrategias cognitivas y metacognitivas (Hernández \& Izquierdo, 2016) que favorezcan la enseñanza de L2, sin que esto signifique cambiar las propiedades de la actividad de la filmación. Experiencia que resulta ser agradable, motivadora, creativa, dinámica y auto reguladora, y que además promueve el trabajo colaborativo y ofrece la oportunidad para explorar nuevos aprendizajes que integra otros campos de conocimiento.

Los resultados obtenidos ratifican que las filmaciones de las prácticas lingüísticas y su respectiva autoevaluación, generaron una mejora de la expresión oral en un $100 \%$ de todos los participantes en comparación con el nivel de inicio. En el caso de los futuros docentes de lengua extranjera, el proyecto «producción de filmes cortos en inglés», además de ser una actividad que potencia el desarrollo de competencias comunicativas, promueve la reflexión personal y del colectivo, propicia el intercambio de opiniones y el trabajo en democracia, fomenta la investigación como una actividad ligada al futuro trabajo profesional, y transfiere a los futuros docentes una estrategia de aprendizaje que muestra ser eficaz.
Con el uso de las tecnologías de apoyo tanto en la estrategia 'producción de videos cortos' como en la estrategia libre basada en la 'repetición de palabras nuevas', todos los participantes del grupo control y del grupo intervención alcanzaron mejores niveles al final del proceso. Sin embargo, los mayores logros se dieron en aquellos participantes que ejecutaron la propuesta didáctica basada en la 'producción de filmes cortos' en idioma inglés. Por tanto, se concluye que el trabajo ejecutado con tecnología de apoyo genera resultados altamente significativos, si es acompañada por una propuesta didáctica que promueva la autovaloración y autocorrección en el momento de usar la lengua extranjera.

\section{Referencias.}

Berk, R. A. (2009). Multimedia teaching with video clips: TV, movies, YouTube, and mtvU in the college classroom. International Journal of Technology in Teaching and Learning, 5(1), 2. in http://desoky.com/ WHY_VIDEOS.pdf

Boyd, D. \& Ellison, N. (2007). Social Network sites: Definition, History, and Scholarship. Journal of Computer-Mediated Communication No. 13(1), 210-230. Recuperado de http://online library.wiley.com/ doi/10.1111/j.1083-6101 .2007.00393.x/epdf.

British Council (2015). English in Ecuador. An examination of policy, perceptions and influencing factors. Disponible en http:// www.obiret-iesalc.udg.mx/es/informes-yestudios/english-ecuador-examinationpolicy-perceptions-and-influencing-factors

Cabero, J. \& Castaño, C. (Coord.) (2009). Herramientas web 2.0 para la formación. Sevilla: Grupo de Investigación Didáctica. Universidad de Sevilla. Recuperado de http:/ 
/www.uhu.es/formacion profesorado/ recursos_academicos/web_2.0/index.htm

Cabero, J., Llorente, M.C., \& Gómez del Castillo, M. (2011). Competencias para

la utilización de las TIC en los procesos de enseñanza-aprendizaje para la implantación del EEES. España: Universidad de Sevilla, Ministerio de Educación. Recuperado de http:// tecnologiaedu.us.es/mec2011/htm/tema9/ presentacion.htm

Cabero, J., \& Marín, V. (2013). Percepciones de los estudiantes universitarios latinoamericanos sobre las redes sociales y el trabajo en grupo. RUSC. Universities and Knowledge Society Journal, vol. 10, núm. 2, julio-diciembre, 2013, pp. 219-235. Disponible en http://www.redalyc.org/pdf/780/ 78028681016.pdf

Cevallos, J., Intriago, E., Villafuerte, J. Molina, G. \& Ortega, L. (2017). Motivation and Autonomy in Learning English as Foreign Language: A Case Study of Ecuadorian College Students. English Language Teaching Journal, 10, (2). Disponible en http://www.ccsenet.org/journal/index.php/ elt/article/view/65784

Esteve, F. (2009). Bolonia y las TIC: de la docencia 1.0 al aprendizaje 2.0. En http:// p o l i r e d.u p m. e s / i n d e x.p h p / lacuestionuniversitaria/article/viewFile/3337/ 3402

Etxebarria, A., Garay, U., \& Romero, A. (2012). Implementation of Social Strategies in Language Learning by Means of Moodle. Journal of Language Teaching and Research. 3, (2), 275-276. DOI: https:// doi.org/10.4304/jltr.3.2.273-282.

Fernández, P. (2009). La competencia ortoépica en el MCER: Crítica a su aplicación en la enseñanza de segundas lenguas. Porta Linguarum, 11, 85-98. En http://www.ugr.es/ $\sim$ portalin/articulos/PL_numero $11 /$ 5\%20P\%20Fernandez $\% 20$ Martin.pdf

Fernández, J.M. \& Torres Gonzáles, J.A. (2015). Teacher attitudes and best practices with ICT faculty Adult Continuing Education in Andalusia. Revista Complutense de Educacion, 26, 33-49. En http:// revistas.ucm.es /index.php/RCED/article/ view/43812/45930, Captured (07, Feb, 2017).

Ferrés, J. \& Piscitelli, A. (2012). La competencia mediática: propuesta articulada de dimensiones. Comunicar, Revista Científica de Comunicación y Educación, 38, 78-79.

García-Salinas, J., Ferreira, A., \& Morales, S. (2012). Autonomía en el aprendizaje de lenguas extranjeras en contextos de enseñanza mediatizados por la tecnología. Revista Onomázein, 25, 15-50. Disponible en http://www.redalyc.org/pdf/1345/ 134524361001.pdf

Garay, U., Luján, C. \& Etxebarria, A. (2013). El empleo de herramientas de la Web 2.0 para el desarrollo de estrategias cognitivas. Porta Linguarum, 20, 169-186. En http://www.ugr.es/ $\sim$ portalin/articulos/PL_numero20/ $11 \% 20 \% 20$ Urtza.pdf

Intriago, E., Villafuerte, J., Morales, M.A., Lema, A. \& Echeverría, J. (2016). Google apps for virtual learning communities development: strengthening English language skills in an university environment. AtoZ: novas práticas em informaçao e conhecimiento, 5 (1), 21-32. DOI: https://doi.org/10.5380/atoz.v5i1.45170

Marco Europeo de Referencia de la Lengua, MCER (2002). Marco Común Europeo, Pp. 99106. En http://cvc.cervantes.es/Ensenanza/ Biblioteca_Ele/marco/cvc_mer.pdf

Mendoza, J. (2015). Otra mirada: la construcción social del conocimiento. Revista POLIS, 11, 1, 83-118. Disponible en http://

- 194 -

Píxel-Bit. Revista de Medios y Educación. No 51 Julio 2017. ISSN: 1133-8482. e-ISSN: 2171-7966. doi: http://dx.doi.org/10.12795/pixelbit.2017.i51.12 
www.scielo.org.mx/pdf/ polis/v11n1/ v11n1a4.pdf, consultado (12.10.2016).

Monteagudo-Valdivia, P. Sánchez, A., \& Hernández, M. (2007). El video como medio de enseñanza. Educ Med Super 21 (2). Disponible en

h t t p : // s c i e 1 o.s ld.cu / scielo.php?script $=$ sci_arttext\&pid $=\mathrm{S} 0864$ 21412007000200006

Quesada, M.J. (2015). Creación de videos educativos como estrategia didáctica para la formación de futuros docentes de inglés. Revista Electrónica "Actualidades Investigativas en Educación», 15(1). Disponible en http://www.scielo.sa.cr/pdf/aie/ v15n1/a06v15n1.pdf

República del Ecuador. (2010). Ley Orgánica de Educación Superior de Ecuador. Disponiblehttp://www.ceaaces.gob.ec/sitio/ wp-content/uploads/2013/10/rloes1.pdf

República del Ecuador. (2011). Ley Orgánica de Educación Intercultural del Ecuador. Disponible en http:// educacion.gob.ec/ley-organica-deeducacion-intercultural-loei/

República de Ecuador, (2015). Sabatina No. 481 de la Presidencia de la República disponible en http://www.elcomercio.com/ actualidad/enlaceciudadano-rafaelcorreamanta-manabi-politica

Ribes, X. (2007). La web 2.0. El valor de los metadatos y de la inteligencia colectiva. TELOS. Cuadernos de Comunicación e Innovación,73. http://www.campusred.net/ $\begin{array}{lllll}\mathrm{T} & \mathrm{E} & \mathrm{L} & \mathrm{O} & \mathrm{S}\end{array}$ articuloperspectiva.asp?idarticulo $=2 \mathrm{yrev}=73$ (Consulta:15.dic/2016)

Sarmiento, E. (2009). Aplicación de las Tics en la Asignatura de Inglés. (Reporte de tesis de grado sin publicar). Universidad Tecnológica Israel.
Saravia, E. \& Bernaus, R. (2008). Motivación y actitudes para el aprendizaje de lenguas de dos colectivos de estudiantes universitarios: futuros maestros de lenguas extranjeras y futuros enfermeros. Porta linguarum 10, 163-184.

Sisto, V. (2012). Análisis del discurso y psicología: a veinte años de la revolución discursiva, Revista de psicología, 21, (1), 185208.

Temprano, A. (2011). Las TIC en la enseñanza bilingüe. Recursos prácticos para la creación de actividades interactivas y motivadoras, Sevilla: Eduforma.

Touré-Tillery, M., \& Fishbach, A. (2014). How to Measure Motivation: A Guide for the Experimental Social Psychologist. Social and Personality Psychology Compass, 8(7), 328341. doi:10.1111/spc3.12110

UNESCO (2008) estándares de competencias en tic para docentes. Recuperado de http://portal.unesco.org/es/ ev.php-URL_ID $=41553$ yURL_DO=DO_TOPI CyURL_SECTION=201.htm

Vera, G. (2012). Introducción de las TIC's en el proceso de enseñanza-aprendizaje de la lengua Castellana. http://vinculando.org/ educacion/introduccion-tic-procesoensenanza-aprendizaje-1enguacastellana.html

Fecha de recepción: 20-12-2016

Fecha de evaluación: 23-02-2017

Fecha de aceptación: 28-03-2017

- 195 -

Píxel-Bit. Revista de Medios y Educación. No 51 Julio 2017. ISSN: 1133-8482. e-ISSN: 2171-7966. doi: http://dx.doi.org/10.12795/pixelbit.2017.i51.12 